\title{
DRUG SAFETY MONITORING IN PATIENTS ATTENDING EPILEPSY CLINIC IN A TERTIARY CARE TEACHING HOSPITAL IN RURAL BENGAL
}

\author{
Sourav Chakrabarty1, Abhijit Das², Ananya Mandal ${ }^{3}$, Samar Biswas ${ }^{4}$, Tanmoy Gangopadhyay5, Avijit Ganguly 6 \\ 1 Post Graduate Trainee, Department of Pharmacology, Bankura Sammilani Medical College, Bankura, West Bengal. \\ ${ }^{2}$ Associate Professor, Department of Pharmacology, Bankura Sammilani Medical College, Bankura, West Bengal. \\ ${ }_{3}^{3}$ Associate Professor, Department of Pharmacology, Bankura Sammilani Medical College, Bankura, West Bengal. \\ ${ }^{4}$ Assistant Professor, Department of Neurology, Bankura Sammilani Medical College, Bankura, West Bengal. \\ ${ }^{5}$ Assistant Professor, Department of Pharmacology, Bankura Sammilani Medical College, Bankura, West Bengal. \\ ${ }_{6}^{6}$ Post-Doctoral Trainee, Department of Pharmacology, Calcutta School of Tropical Medicine, Kolkata.
}

\begin{tabular}{l} 
ABSTRACT \\
\hline BACKGROUND \\
Epilepsy is the second most common neurological disorder affecting fifty million people globally. Antiepileptic Drugs (AEDs) are \\
the mainstay of management in epilepsy. Use of AEDs over prolonged duration makes occurrence of multiple Adverse Drug Reactions \\
(ADRs) frequently, especially with polytherapy.
\end{tabular}

\section{OBJECTIVES}

To estimate the incidence of all the ADRs among patients taking AEDs and to assess their causalities and to quantify their severity.

\section{MATERIAL AND METHODS}

This prospective, observational study was carried out at an Outpatient Referral Epilepsy Clinic at Neurology Department at Bankura Sammilani Medical College, West Bengal, between 1st June and 30th September 2015. The demographic data, diagnosis, drugs prescribed and ADRs experienced by the patients were recorded. Causality and severity assessment was done using Naranjo's Scale and Hartwig's Severity Assessment Scale respectively.

\section{RESULTS}

Incidence of ADRs among the patients who attended the clinic was 3.3\% (105 patients among 3146 experienced at least one ADR). Total 161 ADRs were detected, among which 55.3\% were CNS adverse events followed by $15.5 \%$ gastrointestinal, $14.3 \%$ endocrine, $10.6 \%$ psychiatric abnormalities and $4.3 \%$ related to dermatological and allergic manifestations. Nearly one-third of the ADRs (32.3\%) were found to be possible and 109 (67.7\%) are of probable category, whereas none were deemed to be doubtful or definite. The most commonly implicated suspect drug was valproate (51.5\%) followed by Phenytoin (22.9\%). Most of the ADRs were mild (93.2\%), 5.6\% were moderate and only $1.2 \%$ were deemed severe.

\section{CONCLUSION}

Incidence of ADRs is found to be common in patients on AEDs. Though rare, but they can be life-threatening. Routine safety assessments and pharmacovigilance is necessary in this set up to reduce the incidence and also improve pharmacotherapy and patient compliance.

\section{KEYWORDS}

Antiepileptic drugs, Adverse drug reaction, Pharmacovigilance.

HOW TO CITE THIS ARTICLE: Chakrabarty S, Das A, Mandal A, et al. Drug safety monitoring in patients attending epilepsy clinic in a tertiary care teaching hospital in rural Bengal. J. Evolution Med. Dent. Sci. 2016;5(10):407-413, DOI: 10.14260/jemds/2016/94

\section{INTRODUCTION}

Seizure is a paroxysmal event due to abnormal excessive or synchronous neuronal activity in the brain. ${ }^{1}$ It can be idiopathic or secondary to some other factors like genetic, congenital CNS abnormality, metabolic, vascular, infection, trauma, neoplasms, degenerative disease, etc.

Financial or Other, Competing Interest: None.

Submission 21-12-2015, Peer Review 13-01-2016,

Acceptance 22-01-2016, Published 02-02-2016.

Corresponding Author:

Dr. Avijit Ganguly,

BG 116, Rabindrapally,

Krishnapur, P.O. Prafullakanan,

Kolkata-700101.

E-mail: avijitdec81@gmail.com

DOI:10.14260/jemds/2016/94
A $5-10 \%$ of the population have at least one seizure in their lifetime. ${ }^{1}$ But the term 'Epilepsy' must be distinguished from seizure. In 2014, International League Against Epilepsy (ILAE) has defined epilepsy "as a condition with two or more unprovoked seizures occurring more than $24 \mathrm{hrs}$. apart." 2 It is one of the oldest condition with reference found dating back to 4000 BC. ${ }^{3}$ Among neurological disorders, it has the second highest prevalence after stroke and it affects around fifty million people globally, whereas $80 \%$ of them live in low and middle income countries. ${ }^{3}$

It accounts for $0.75 \%$ of global burden for disease. ${ }^{3}$ In India, there are 10 million people living with epilepsy. 4 Prevalence in rural areas (11.9/1000 population) is twice as much as that of urban areas (5.7/1000 population) according to Bangalore Urban Rural Neuro-epidemiological Survey (BURNS). ${ }^{4}$ 
A percentage of these patients with active epilepsy is not on treatment or on inadequate treatment, which correspond to the term "Treatment gap."

Medical management with Anti-Epileptic Drugs (AED) are the mainstay of management of epilepsy, both effective and affordable even in developing countries. Currently, we have 7 traditional AEDs, which were introduced before 1990 and at least 17 newer AEDs, which were introduced after that period. Selection of AEDs is done on the basis of seizure type, comorbidities, cost, pregnancy/lactation, occupation, etc. Inadequate seizure control may lead to opting for add-on therapy, which is usually done by a trial and error basis.

Adverse Drug Reaction as defined by WHO is "Any response to a drug, which is noxious and unintended and which occurs at doses normally used in man for prophylaxis, diagnosis or therapy of disease or for the modification of physiological functions." 5 AEDs, specially the conventional ones have significant adverse effects on the recipients. In epilepsy, most of them have to be taken for a longer period (Minimum 3 years), which leads to significant ADRs. They also have significant drug interactions in between themselves when given together.

This is one of the reasons of discontinuation of treatment, non-compliance or treatment gap. Occurrence of ADRs depend on age, sex, ethnicity, socioeconomic status, dose and rate of initiation of AEDs, etc. A large proportion of primary seizure patients are of childhood and early adulthood ages and the secondary seizures occurring in elderly ages. There is also a number of women of reproductive age groups presenting with epilepsy with adverse effect on pregnancy, lactation and contraception. All these groups are vulnerable for experiencing ADRs with antiepileptic drugs.

Though there are some published studies on ADR monitoring in patients with epilepsy, both worldwide and in India, i.e. Bangalore, Kolkata, etc.8-12 However, we have not found much data on safety profile of AEDs in patients from rural areas. In these areas a large proportion of patients belong to tribal communities who have unique pharmacogenetic profiles. Hence, we decided to undertake this study in Bankura Sammilani Medical College, which is a Tertiary Care Teaching Hospital in rural Bengal.

\section{AIMS AND OBJECTIVES}

The aim of this study was to estimate the incidence of all the ADRs among patients taking AEDs and to assess the causalities of ADRs on the basis of Naranjo's adverse drug reaction probability scale. ${ }^{6}$ We also attempted to quantify the severity of ADRs on the basis of Hartwig's severity assessment scale. ${ }^{7}$

\section{MATERIALS AND METHODS}

This was a prospective, observational study performed at the Epilepsy Clinic at the Department of Neurology, Bankura Sammilani Medical College, Bankura, West Bengal, for a duration of 4 months starting from 1st June to 30th September 2015. As per inclusion criteria, we enrolled all the patients diagnosed by a consultant neurologist as having seizure (Primary/secondary) and prescribed at least one antiepileptic drug. As per exclusion criteria, those having comorbidities other than those related to seizure disorders were excluded. Demographic variables, diagnosis, AEDs prescribed were noted separately. Only those either spontaneously reported a suspected ADR or were diagnosed by a neurologist during routine examination were further evaluated by pharmacologists.

Detailed clinical and drug history and relevant information about the suspected reaction, its onset, duration, severity, temporal association with the drug if any, past history, co-morbidities, concomitant medication, relevant laboratory investigations were collected and thoroughly analysed to reach at a clinical diagnosis. All informations were collected in an ADR reporting form. Further the reactions were categorized and causalities were assessed by Naranjo's ADR probability scale and severity was quantified by Hartwig's severity assessment scale. All the data were compiled in Microsoft Excel datasheet and statistical analysis was done using tools of descriptive statistics and chi-square test with the help of SPSS 22 and EPICALC 2000. A P value less than 0.05 was set to be taken as significant. Prior approval of Institutional Ethics Committee was taken and informed consent was taken from the patients or their legal representatives.

\section{RESULT}

Total 3146 patients were prescribed at least one AED during the entire period of study, among which $52.7 \%$ were females. The age distribution of the patients are given in Table 1.

\begin{tabular}{|c|c|}
\hline Age Group (Yrs) & Percentage of Total Patients \\
\hline $0-5$ & $11.1 \%$ \\
\hline $6-20$ & $56.5 \%$ \\
\hline $21-35$ & $10.4 \%$ \\
\hline $36-50$ & $6.4 \%$ \\
\hline $51-65$ & $9.1 \%$ \\
\hline$>65$ & $6.5 \%$ \\
\hline \multicolumn{2}{|c|}{ Table 1: Age Distribution of Patients } \\
\hline
\end{tabular}

As per diagnosis by consultant neurologist, primary seizure was more common $(65 \%)$ than the secondary ones (35\%). While collecting and analysing the data regarding the use of AEDs in patients we found that monotherapy is the most common (67\%) regimen, while in $28.3 \%$ of patients two AEDs have been used and only in $4.7 \%$ cases three or more drugs have been used. Valproate monotherapy has been most prevalent (36.3\%) followed by phenytoin monotherapy (14.6\%). The pattern of use of AEDs can be shown in Figure 1. 


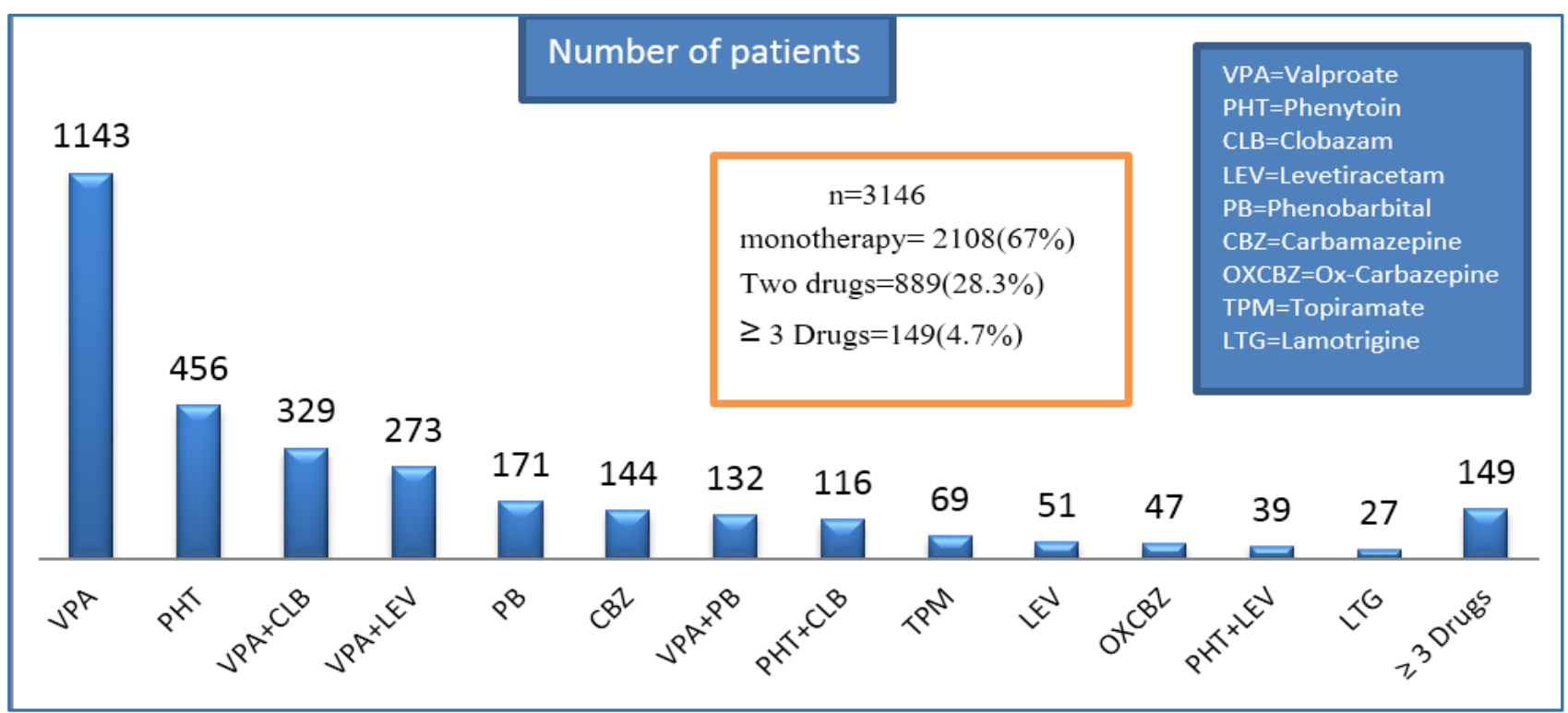

Fig 1: Pattern of Antiepileptic drug use

Now out of these 3146 patients, only 105 patients suffered from at least one suspected Adverse Drug Reaction (ADR) with incidence rate of 3.3\%. Total 161 ADRs were found with a mean of 1.53 ADR per case. The mean age of cases who experienced the ADRs were $21.54 \pm 4.364$ years with a female preponderance of $55.3 \%$. The system wise classification of the ADRs experienced are summarised in Figure 2.

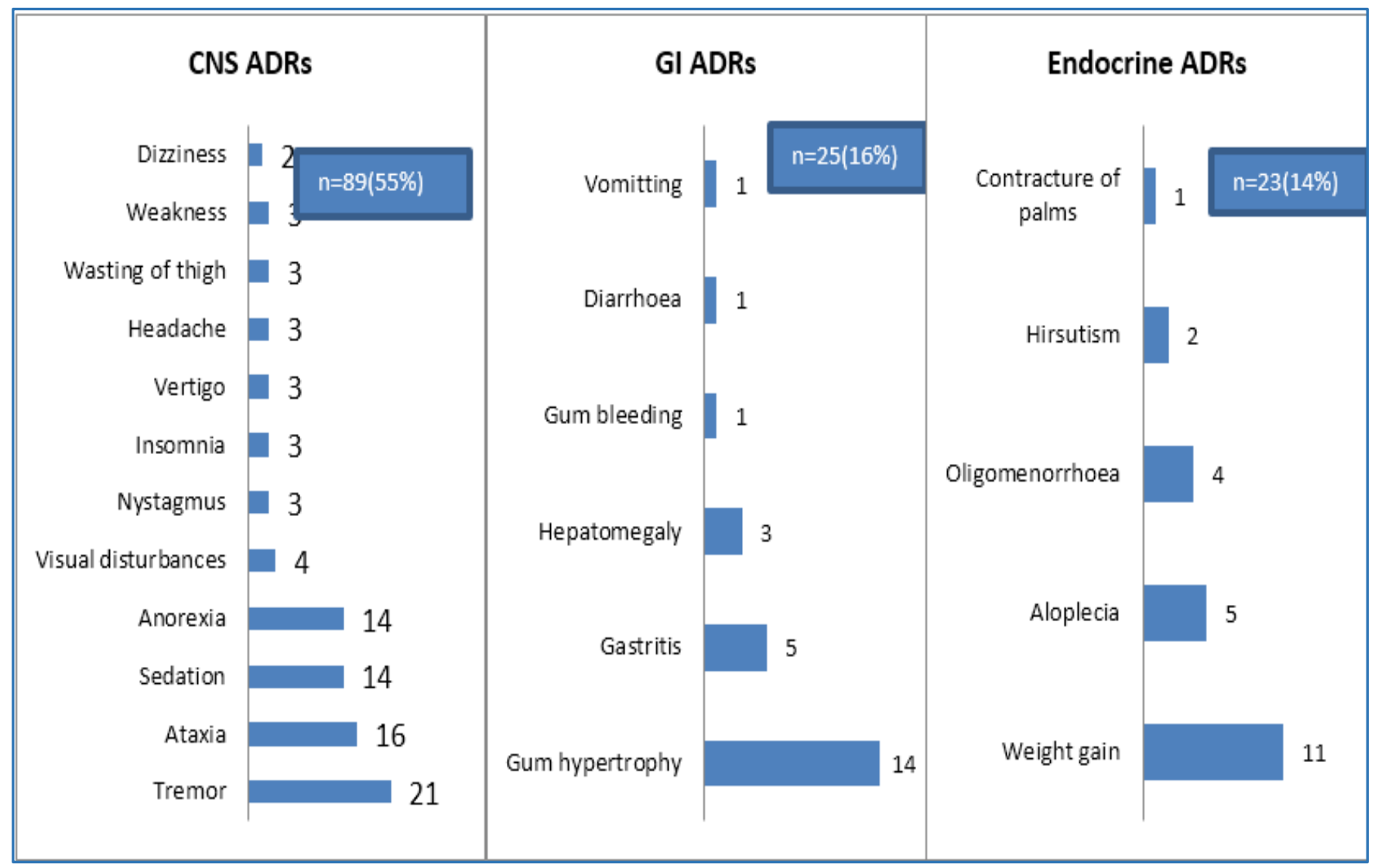

Fig 2: Distribution of adverse drug reactions as per different organ systems involved 


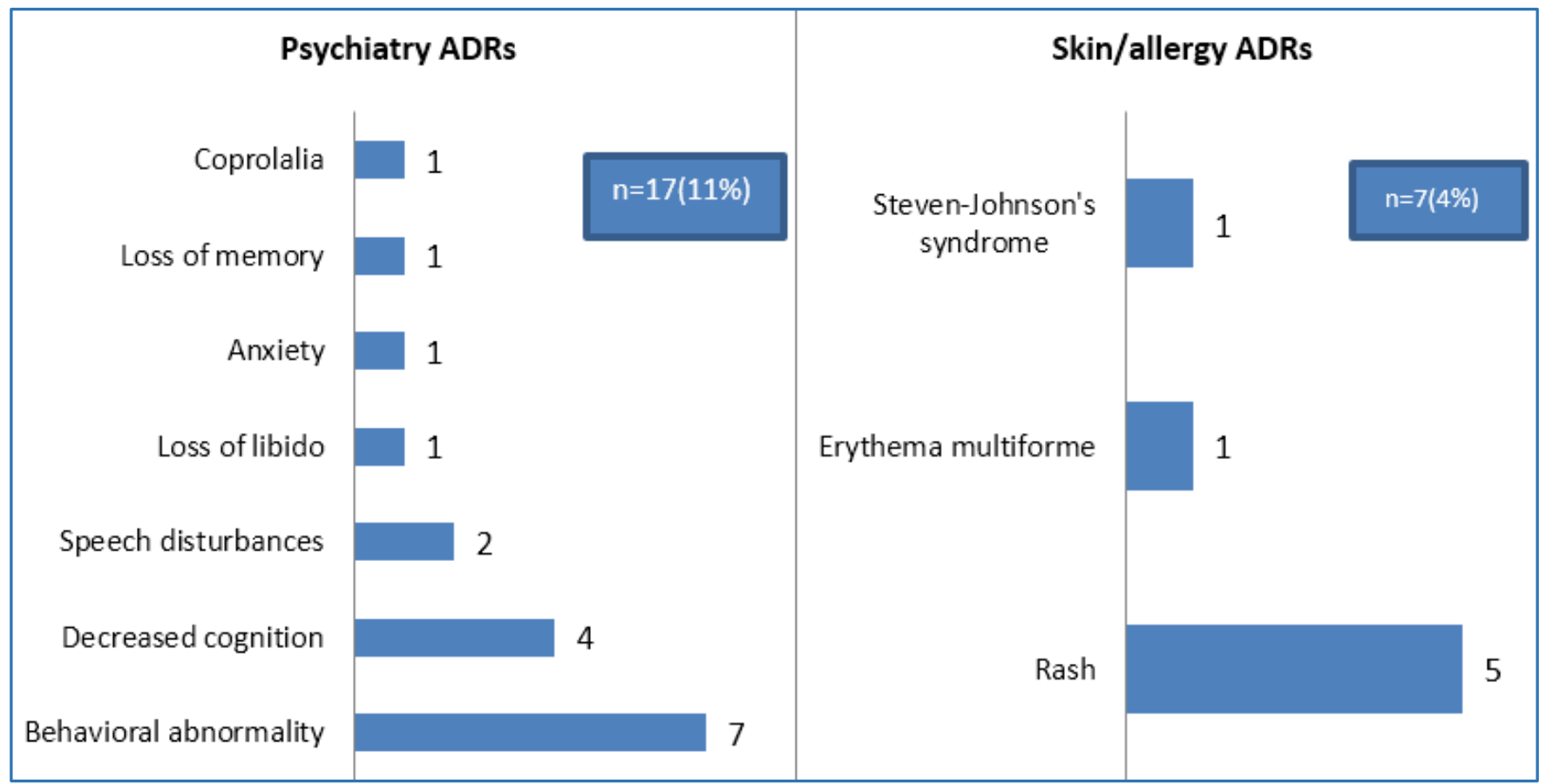

Thorough analysis of the ADR data revealed that tremor was the most common ADR found affecting 21 cases, 17 of which is suspected to be caused by valproate alone. The other most common ADRs are in order of decreasing frequency was ataxia (16), sedation (14), anorexia (14), gum hypertrophy (14) and weight gain (11). Occurrence of these ADRs mostly led to dose modification or de-challenge of the suspected drug. While assessing the causality of the ADRs, we found that valproate was the most common suspect drug accounting for 51.5\% ADRs followed by Phenytoin (22.9\%) and Carbamazepine (8.1\%). The detailed analysis of ADR causalities reveals the Figure 3.

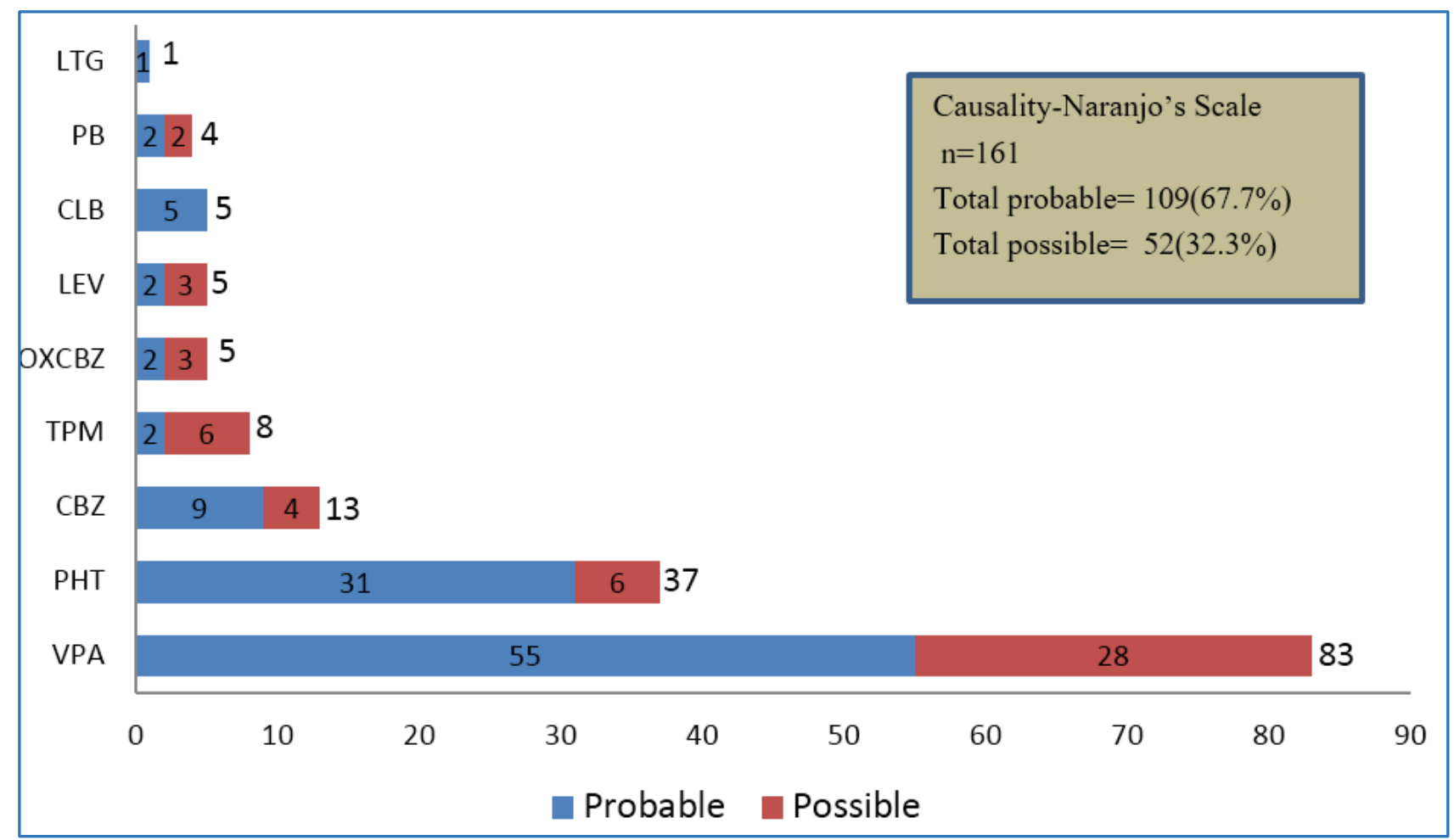

Fig 3: Causality assessment of different antiepileptic drugs used

The Figure 3 shows that total 52 ADRs (32.3\%) were found to be of possible category and 109 (67.7\%) were of probable category according to Naranjo's ADR probability scale. None of the ADRs were found to be doubtful or definite category. As the total number of drugs taken differs widely in each group, so only the numbers of ADRs in every groups are not ideally comparable. Hence, we have further modified the data by finding out the percentage of incidence of ADRs in each drug group and the result obtained is shown in Figure 4. 


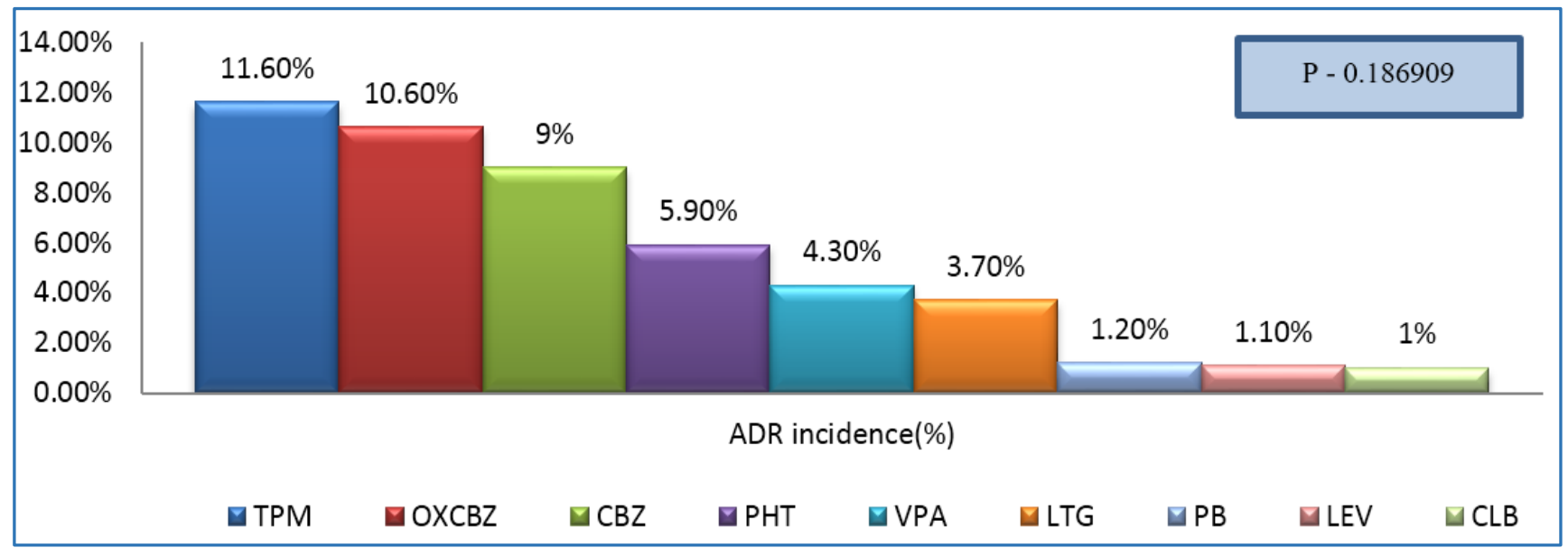

Fig 4: ADR incidence with different antiepileptic drugs

Strikingly, we found that though valproate is suspected to cause most number of ADRs, but incidence per 100 recipients is only $4.3 \%$, far below than that of Topiramate $(11.6 \%)$, Oxcarbamazepine (10.6\%), Carbamazepine (9\%) and Phenytoin (5.9\%). Newer AEDs like Clobazam (1\%), Levetiracetam $(1.1 \%)$ and lamotrigine $(3.7 \%)$ were found to be safer, while some conventional AED like phenobarbitone was found to cause less percentage of ADRs (1.2\%). While comparing the ADR incidence according to regimen, we found the data as shown in the Tables 2-4.

\begin{tabular}{|c|c|c|c|}
\hline Regimen & Intake & $\begin{array}{c}\text { ADR } \\
\text { Incidence } \\
\text { (\%) }\end{array}$ & $\begin{array}{c}\text { P value } \\
\text { (chi- } \\
\text { square) }\end{array}$ \\
\hline Conventional & 3045 & $4.49 \%$ & \multirow{2}{*}{0.000230} \\
\cline { 1 - 2 } Newer & 1131 & $2.03 \%$ & \\
\hline \multicolumn{3}{|c|}{ Table 2: Difference between ADRs with conventional } \\
antiepileptic drugs and newer agents \\
\hline
\end{tabular}

\begin{tabular}{|c|c|c|c|}
\hline Regimen & Intake & $\begin{array}{c}\text { ADR Incidence } \\
\text { (\%) }\end{array}$ & $\begin{array}{c}\text { P value } \\
\text { (chi- } \\
\text { square) }\end{array}$ \\
\hline Monotherapy & 2108 & $4.22 \%$ & 0.001177 \\
\cline { 1 - 2 } Polytherapy & 1038 & $6.93 \%$ & \\
\cline { 1 - 2 } Table 3: Difference between ADRs in \\
monotherapy and polytherapy groups
\end{tabular}

\begin{tabular}{|c|c|c|c|}
\hline Gender & Intake & $\begin{array}{c}\text { ADR Incidence } \\
\text { (\%) }\end{array}$ & $\begin{array}{c}\text { P value } \\
\text { (chi- } \\
\text { square) }\end{array}$ \\
\hline Male & 1487 & $4.84 \%$ & 0.50869 \\
\hline Female & 1659 & $5.36 \%$ & \\
\cline { 1 - 1 } & \multicolumn{3}{|c|}{$\begin{array}{c}\text { Table 4: Difference between ADRs } \\
\text { among males and females }\end{array}$} \\
\hline
\end{tabular}

The tables show that the percentage of ADR incidence is significantly higher in conventional drugs vs newer AEDs, so as the polytherapy vs monotherapy. But the gender distribution of patients does impart any significant difference in incidence percentages.

While trying to quantify the severity of ADRs as per Hartwig's severity assessment scale, we found that 150 ADRs $(93.2 \%)$ were of mild category, whereas total 9 cases (5.6\%) were deemed to be of moderate category. Results are shown in Figure 5.

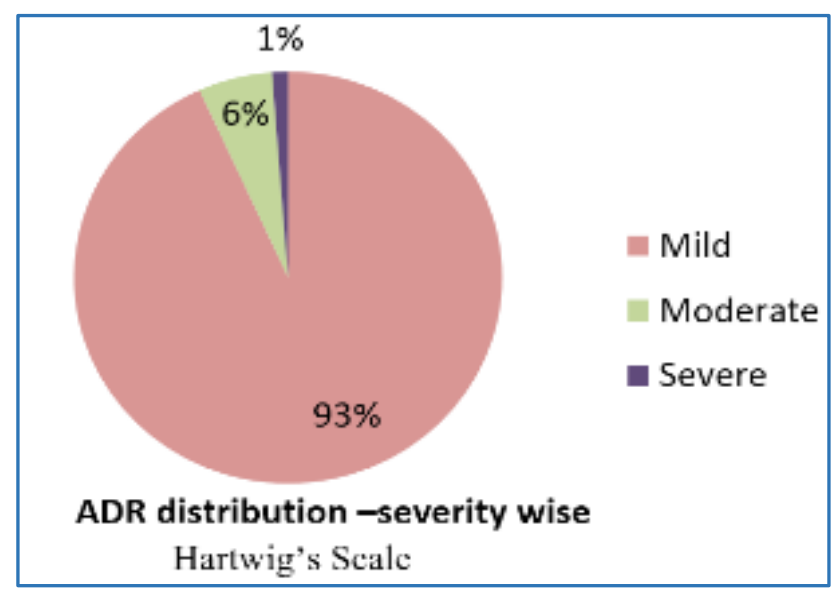

\section{Fig 5: Distribution of ADRs according to severity}

Only two cases were of severe category, one case of Steven Johnson's Syndrome (SJS) by Phenytoin sodium and another case of Erythema Multiforme (EM) by valproate, where patients required hospital admission and intensive monitoring. Photographs were taken from the patient of Steven Johnson's Syndrome (SJS) after obtaining written consent from the patient both at the beginning of ADR and after 7 days of withdrawal of drugs. The picture is shown in Figure 6.

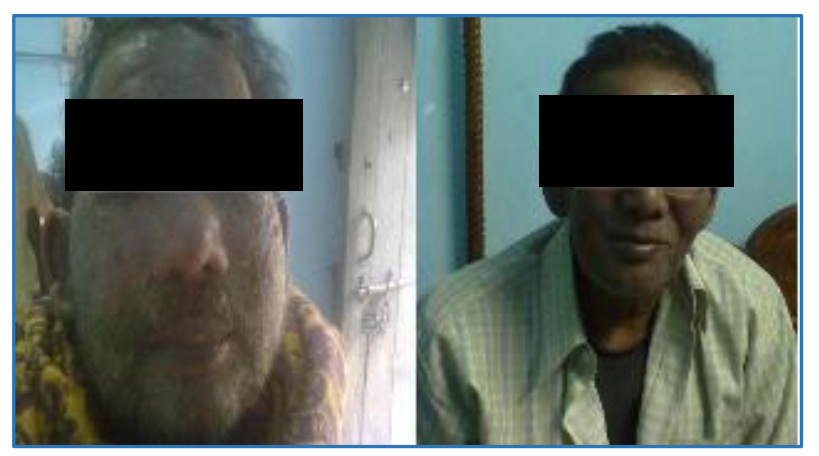

Two days after start of SJS

7 days after withdrawal of suspect drug (Phenytoin)

Fig. 6: The picture of Steven Johnson Syndrome before and after withdrawal of Phenytoin 
It can be concluded that none of the ADRs resulted any permanent damage or death of the patients.

\section{DISCUSSION}

This prospective observational study was undertaken for assessment of incidence of ADRs among epilepsy patients, detecting the most suspect drugs for those ADRs and also for quantifying severity of the ADRs followed by necessary action to be taken. We found some similar studies done in recent years and compared our results with them.

Narayan et al. ${ }^{8}$ conducted a retrospective study in Neurology Department, Bangalore, in 2008. In 788 patients, they found ADRs in 80 patients (Incidence 10.27\%). ADR incidence was $9.18 \%$ in monotherapy and $11.56 \%$ in polytherapy. However, the chi-square test yielded no statistically significant difference between monotherapy vs polytherapy. Phenytoin was most common suspected drug (14.28\%) followed by clobazam (12.5\%). Maximum ADRs were moderate $(70.83 \%)$, whereas $25 \%$ were severe in category according to WHO scale. A $65.62 \%$ ADRs were probable, $13.5 \%$ possible and $20.8 \%$ were definite also at WHO causality assessment scale. Hence, the incidence of ADRs was greater than that of our study. The probable reason may be that we mainly relied upon the information given spontaneously by the patients or by clinical assessment by the neurologist. If we performed relevant laboratory investigations, incidence could be higher.

Palanisamy et al. ${ }^{9}$ in 2009 found in their study that among 268 patients only 16 patients had ADRs with incidence of $5.9 \%$. Rash was the most common ADR, whereas Phenytoin was the most common suspect drug accounting for $50 \%$ of ADRs, mainly involving rash, ataxia, Steven Johnson's Syndrome, etc. Similar ADRs have been found in our study also. Carbamazepine and Oxcarbazepine resulted ADRs in 4 patients each. Though Valproate was the most commonly used AED, it only resulted in one ADR (Rash).

Pal et al. ${ }^{10}$ found that ADR incidence was $16.28 \%$ in their study. Total 86 patients had ADRs, $52.48 \%$ of them were possible and $25.58 \%$ were probable. Most frequently involved system was CNS ADRs (34.9\%), similar to our study. Majority of ADRs were mild (39.53\%). Rate of occurrence of ADRs among monotherapy vs polytherapy was $12 \%$ and $17.28 \%$ respectively. Females had a more susceptibility (55.8\%) than males (44.2\%).

Ramkrishna et al. ${ }^{11}$ found that out of 2880 patients treated with AEDs, only 85 patients (2.95\%) experienced ADR. Again CNS ADRs were most common, whereas ataxia was leading CNS ADR. A 59.7\% of ADRs were Type A, followed by Type C (27.61\%). According to Naranjo's scale $87.3 \%$ were probable with rest classified as possible. Maximum ADRs were mild (48.5\%). Here majority of the ADR occurred among males. Phenytoin was found to be most offending drug followed by valproate.

We found from our study that AED usage pattern at our Epilepsy Clinic favours monotherapy. ADR incidence in our study was less than some contemporary studies. This is probably due to three reasons: First, most of our study patients belonged to younger age groups with less proportion of extreme age groups, who are most susceptible to ADRs. This may be due to reasons that most of the paediatric age group patient were taken to the paediatric OPD instead of Neurology OPD, whereas elderly patients were mostly with secondary seizures who visited medicine or surgery OPD/Indoor. Secondly, the study period was relatively short and insufficient to measure the long-term ADRs of anti-epileptic therapy. Third, the use of laboratory investigations was very limited in our study for the unwillingness on the part of the patients for adequate follow-up.

We found that majority of ADR were CNS ADR, owing to the principal site of action of AEDs. A two-third of ADRs were probable while rest were possible. Maximum of ADRs were mild in severity. Serious adverse event was $1.2 \%$, though there was not any mortality. There was statistically significant difference in ADR incidence between individual drugs and gender distribution. But there was significant difference in ADR incidence between conventional vs newer drugs and monotherapy vs polytherapy.

Like all studies, our study also had some limitations. First, we did not take into consideration the dose of the drugs, which is a major factor in occurrence of ADRs. Secondly, we did not measure the serum concentration of drugs to find out any association with the ADR occurrence.

\section{CONCLUSION}

To conclude adverse drug reactions are more common with antiepileptic therapy, especially with polypharmacy. However, serious ADRs were not significant in our study. We also think that rational prescription supports monotherapy from this study. In future we expect that this study will motivate the health care professionals in the Department of Neurology, BSMCH, to report ADRs spontaneously for improving pharmacotherapy and patient compliance. Lastly, we conclude that more phase IV trials should be conducted, especially with newer AEDs to know their actual safety and tolerability.

\section{REFERENCES}

1. Kasper DL, Fauci AS, Hauser SL, et al., editors. Harrison's principles of internal medicine. 19th ed. New York: McGraw Hill; 2015.

2. International League Against Epilepsy. Available from: http://www.ilae.org/Visitors/Centre/Definition.cfm.

3. World Health Organization [Internet]. Geneva: World Health Organization 2015 [cited Dec 2015]. Available from:

http://www.who.int/mediacentre/factsheets/Epilepsy May 2015.

4. Satishchandra P, Sinha S, Santhosh NS. Epilepsy: Indian Perspective. Ann Indian Acad Neurol. 2014 Mar;17 (Suppl 1):s3-s11.

5. Srinivasan R, Ramya G. Adverse drug eruption-Causality assessment. Int J Res Pharm Chem 2011;1(3):606-12.

6. Naranjo CA, Busto U, Sellers EM, et al. A method for estimating the probability of adverse drug reactions. Clin Pharmacol Ther 1981;30:239-45.

7. Hartwig SC, Siegel J, Schneider PJ. Preventability and severity assessment in reporting adverse drug reactions. A J Hosp Pharm 1992;49:2229-31.

8. Roopa BS, Narayan SS, Sharma GRK, et al. Pattern of adverse drug reactions to anti-epileptic drugs: a crosssectional one-year survey at a Tertiary Care Hospital. Pharmacoepidem. Drug Safety. 2008 Jan; 17(8):807-12. 
9. Arulkumaran KSG, Palanisamy S. A study on drug use: evaluation of anti-epileptics at a Multi-speciality Tertiary Care Teaching Hospital. Int J Pharm Tech Res 2009;1(4):1541-47.

10. Pal A, Prusty SK, Sahu PK, et al. Drug utilization pattern of anti-epileptic drugs: a pharmacoepidemiologic and pharmacovigilance study in a Tertiary Teaching Hospital in India. Asian J Pharm Cln Res 2011;4(1):96-99.
11. Ramakrishna P, Ramaiah M, Kayal AK, et al. Collection, detection, assessment, monitoring and prevention of adverse drug reactions of anti-epileptic drug. Int Res J Pham 2014;5(8):653-57.

12. Jena M, Jena SS, Dash M. Monitoring of prescriptions and pharmacovigilance evaluation of anti-epileptic drugs in a Tertiary Care Teaching Hospital. Int J Pharm Sci Rev Res. 2014 Nov; 29(1):99-104. 